\title{
Erratum to: Clinicopathological evaluation of cyclooxygenase-2 expression in meningioma: immunohistochemical analysis of 76 cases of low and high-grade meningioma
}

\author{
Yasutaka Kato $\cdot$ Hiroshi Nishihara $\cdot$ Hiromi Mohri • \\ Hiromi Kanno $\cdot$ Hiroyuki Kobayashi · Taichi Kimura • \\ Mishie Tanino $\cdot$ Shunsuke Terasaka $\cdot$ Shinya Tanaka
}

Published online: 19 March 2013

(C) The Japan Society of Brain Tumor Pathology 2013

\section{Erratum to: Brain Tumor Pathol}

DOI 10.1007/s10014-012-0127-8

1. Fig. 2e:

The authors have noted some errors in the original article publication. In Fig. 2e, figure legend and the text (p5, line 3) the $R^{2}$ value has been published inadvertently.

The corrected figure and text should appear as given below.

The online version of the original article can be found under doi:10.1007/s10014-012-0127-8.

Y. Kato · H. Kanno - T. Kimura - M. Tanino - S. Tanaka Department of Cancer Pathology, Hokkaido University,

Graduate School of Medicine, Sapporo, Japan

H. Nishihara $(\square)$

Laboratory of Translational Pathology, Hokkaido University

School of Medicine, North 15, West 7,

Kita-ku, Sapporo 060-8638, Japan

e-mail: hnishihara@s5.dion.ne.jp

H. Mohri

Laboratory of Oncology, Hokuto Hospital, Obihiro, Japan

H. Kobayashi · S. Terasaka

Department of Neurosurgery, Hokkaido University,

Graduate School of Medicine, Sapporo, Japan

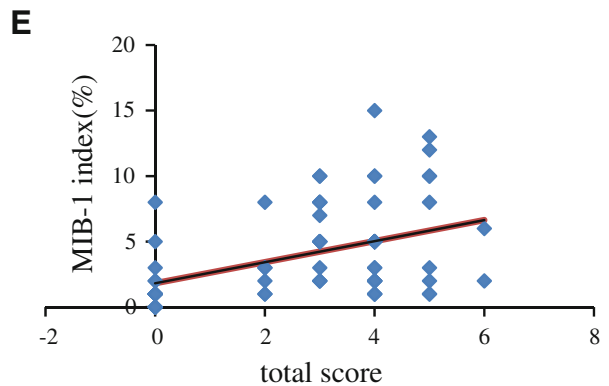

2. In figure legend for Fig. 2

There is a statistically strong relationship between COX-2 expression level and MIB-1 index.

3. Text (p5, line 3)

Scatter plot analysis revealed a significant relationship between COX-2 expression level and MIB-1 labeling index (Fig. 2e). 